\title{
Anabases
}

ANABASES Traditions et réceptions de l'Antiquité

$26 \mid 2017$

Varia

\section{Les références antiques des fondateurs de l'Amérique française au XVIIe siècle}

Nicolas Faelli

\section{OpenEdition}

1 Journals

Édition électronique

URL : http://journals.openedition.org/anabases/6198

DOI : 10.4000/anabases.6198

ISSN : 2256-9421

Éditeur

E.R.A.S.M.E.

Édition imprimée

Date de publication : 1 novembre 2017

Pagination : 19-32

ISSN : 1774-4296

\section{Référence électronique}

Nicolas Faelli, «Les références antiques des fondateurs de l'Amérique française au XVIle siècle », Anabases [En ligne], 26 | 2017, mis en ligne le 01 novembre 2020, consulté le 20 janvier 2021. URL : http:// journals.openedition.org/anabases/6198 ; DOI : https://doi.org/10.4000/anabases.6198

(c) Anabases 


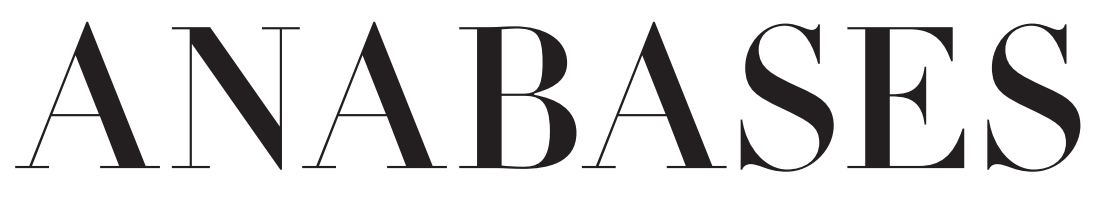

Traditions et Réceptions de l'Antiquité

$$
\begin{aligned}
& N \circ 26 \\
& 2017
\end{aligned}
$$

\section{E.R.A.S.M.E.}

Université Toulouse - Jean Jaurès 



\section{Sommaire}

$\mathrm{N}^{\circ} 26-2017$

\section{Historiographie et identités culturelles}

Jocelyne Peigney

La polysémie poétique d'anabase chez Saint-John Perse :

évocations, étymologie et botanique $\ldots \ldots \ldots \ldots \ldots \ldots \ldots \ldots \ldots \ldots$

Nicolas FAELLI

Les références antiques des fondateurs de l'Amérique française

au XVII siècle ................................. I9

Silvia Alaura

Setting the stage for Hittite studies in Victorian England:

practices and methods of the 1870 . . . . . . . . . . . . .

\section{Traditions du patrimoine antique}

“ Restituer Herculanum I. Des archives de fouilles aux restitutions 3D »

Alexandra Dardenay et Adeline Grand-Clément

Restituer Herculanum au XXI ${ }^{\mathrm{e}}$ siècle. L'apport des archives. . . . . . . . 55

Antonella Coralini

In situ e alibi, dallo scavo integrato alla cultura dell'abitare :

Vesuviana-DHER a Ercolano . . . . . . . . . . . . . . . . 67 67

Agnes Allroggen-Bedel

I documenti settecenteschi come strumenti per lo studio

degli scavi ercolanesi . . . . . . . . . . . . . . . Io3

James ANDREws

Revisiting the upper floors of the casa di Argo at Herculaneum . . . . . . II7 
Nicolas MonteIx

Les archives des fouilles d'Herculanum au XX $\mathrm{XX}^{\mathrm{e}}$ siècle :

analyse critique et pistes d'exploitation . . . . . . . . . . I43

\section{Archéologie des savoirs}

Yannick Le PAPE

L'inspiration et l'imprudence. Poésie de l'anticomanie

dans la critique d'art du second XIX ${ }^{\mathrm{e}}$ siècle . . . . . . . . . . . . . I57

\section{Actualités et débats}

Claude AzızA

Antiquités parallèles (7). La starlette des bandelettes . . . . . . . . . I77

Alexandra TrachseL

La réception d'Homère dans le domaine de la rhétorique $\ldots \ldots \ldots$. . . I83

Claude Aziza

Curiosa (2) In vino (vin/eau) veritas (very tasse) : drame de l'ivresse . . . . I89

\section{Lire, relire la bibliothèque des sciences de l'Antiquité}

Laurent OLIVIER

Pour en finir avec la race : Henri Hubert et l'anthroposociologie

À propos du commentaire de “L'Aryen, son rôle social », de Vacher de Lapouge $(\mathrm{r} 899) \ldots \ldots \ldots$

Henri Hubert

Compte rendu de Georges VACher de LAPouge, L'Aryen, son rôle social, L'Année sociologique, igoo, p. i45-146 . . . . . . . . . . . . . . . . . 203

\section{L'atelier de l'histoire : chantiers historiographiques}

L'Atelier des doctorants (coordonné par Adeline Grand-Clément) (14)

Francesco LoDÀ,

L'initiation des guerriers chez les peuples indo-européens.

Une enquête historiographique dans les pas de Georges Dumézil . . . 207

\section{Comptes rendus}

Benjamin Anderson et Robert G. Ousterhout

Palmyra I885. The Wolfe Expedition and the photographs

of John Henry Haynes (M. Sartre) . . . . . . . . . . . . . . . . . . . 2 215 
Mary R. BAchvarova

From Hittite to Homer. The Anatolian Background of Ancient Greek Epic (G. Hoffmann) . . . . . . . . . . . . . . . . .

Patrick Baker, Ronny Kaiser, Maike Priesterjahn, Johannes Helmrath (éd.) Portraying the Prince in the Renaissance. The Humanist Depiction of Rulers in Historiographical and Biographical Texts (G. Demelemestre). . 220

Brigitte BAKHоUсHE (éd.)

Science et exégèse. Les interprétations antiques et médiévales du récit biblique de la création des éléments (Genèse I, I-8) (F. P. BARONE) . . . . . . .

Laurence Baurain-Rebillard (éd.)

Héros grecs à travers le temps : autour de Persée, Thésée, Cadmos et Bellérophon (C. Giovénal) . . . . . . . . . . . . . . . .

Mark W. PAdiLla,

Classical myth in four films of Alfred Hitchcock . . . . . . . . . . . . . . .

Vivien Bessieres

Le Péplum et après? L’Antiquité gréco-romaine dans les récits contemporains (Cl. Aziza) . . . . . . . . . . . . . . . . . 225

Pierre Briant

Alexandre. Exégèse des lieux communs (É. Guillon) . . . . . . . . . . . . . 227

Paulo ButTi DE Lima

Il piacere delle Immagini. Un tema aristotelico nella riflessione moderna sull'arte (C. Noacco) . . . . . . . . . . . . . . . . . . .

Bénédicte Coudì̀re

La truelle et le phylactère. La proximité des images (M. Scapin) . . . . . . . 23I

Thorsten Fögen, Richard WARREN (éd.)

Graeco-Roman Antiquity and the Idea of Nationalism in the rgth Century, Case Studies (M. Champier) . . . . . . . . . . . . . . . ${ }_{232}$

Ronald H. Fritze

Egyptomania. A History of Fascination, Obsession and Fantasy

(Fabien Bièvre-Perrin) . . . . . . . . . . . . . . . . . . . . . 234

Joseph Geiger

Hellenism in the East. Studies on Greek Intellectuals in Palestine

(C. Bonnet). . . . . . . . . . . . . . . . . 236

Tiffany Jenkins

Keeping their marbles. How the treasures of the past ended up in museums... and why they should stay there (C. Valenti) . . . . . . . . . . 238 
Ayelet H. Lushkov

Magistracy and the Historiography of the Roman Republic.

Politics in Prose (C. Landrea) . . . . . . . . . . . . . . . . . . . . . . 240

Jean-Claude MüHLEthaler

Énée le mal-aimé. Du roman médiéval à la bande dessinée

(Fl. Bouchet) ........................ 24I

Laurent PERNoT

Epideictic Rhetoric, Questioning the Stakes of Ancient Praise

(C.Psilakis) ......................... 243

Priscian

Answers to King Khosroes of Persia (O. Gengler) . . . . . . . . . . . . . . 245

Paul Robertson

Paul's letters and contemporary Greco-Roman literature:

theorizing a new taxonomy (M. Cambron-Goulet) . . . . . . . . . . . . . . 247

Federico Santangelo (éd.)

Approaching the Roman Revolution. Sir Ronald Syme,

Papers on Republican History (C. Landrea) . . . . . . . . . . . . . . . . . . 249

Molly Swetnam-BurLand

Egypt in Italy. Visions of Egypt in Roman Imperial Culture

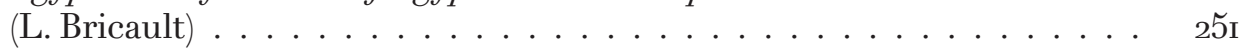

Helen VAN Noorden

Playing Hesiod. The 'Myth of the Races' in Classical Antiquity

(D. Bonanno)

Résumés . . . . . . . . . . . . . . . . . . . . . . . . . . . . . . . 257

Index .......................... . . 265 


\section{Historiographie et identités culturelles}





\section{Les références antiques des fondateurs de l'Amérique française au XVIIe siècle}

Nicolas FAELLI

D epuis le début des Temps modernes, il existe en France un rapport entre l'Histoire, la mythologie gréco-latine et la propagande d'État ${ }^{1}$. Cette mode littéraire connut son paroxysme au XVII ${ }^{\mathrm{e}}$ siècle avec l'avènement d'une monarchie très centralisée qui essayait de s'inscrire dans la continuité d'un passé glorieux. Des auteurs comme Chantal Grell ou Pierre Briant ont ainsi analysé la place de l'Antiquité, et surtout de l'histoire d'Alexandre le Grand ${ }^{2}$, dans la propagande de Louis XIII et Louis XIV. Outre son utilisation par les autorités elles-mêmes, l'exaltation de la grandeur de l'État passait par une récupération de l'Antiquité chez la plupart des auteurs qui cherchaient à mettre en valeur leur nation ${ }^{3}$. Si au début du XVII ${ }^{e}$ siècle peu de gens en France connaissaient le grec, la poésie homérique connaissait depuis quelques décennies un regain d'intérêt, notamment grâce à une première traduction en français de l'Odyssée en $1547^{4}$.

1 Notamment sous les règnes d'Henri II et Charles XI, sous la plume d'auteurs comme Pierre de Ronsard ou Étienne Jodelle. Fr. Joukovsкy-місна, Poésie et mythologie au XVI siècle, Paris, Nizet, 1969, p. 141-160.

2 Ch. Grell, Histoire intellectuelle et culturelle de la France du Grand Siècle (1654-1715), Paris, Nathan, 2000 ; EAD., "Les historiographes en France XVI ${ }^{\mathrm{e}}$ XVIII ${ }^{\mathrm{e}}$ siècles ", in Ch. Grell (dir.), Les Historiographes en Europe de la fin du Moyen Âge à la Révolution, Paris, PUPS, 2006, p, 127-156 et P. Briant, Alexandre des Lumières. Fragments d'Histoire européenne, Paris, Gallimard, 2012.

3 Voir P. Burke, "Exemplarity and anti-exemplarity in early modern Europe », in S. Hodkinson \& T. D. Barnes (dir.), Sparta. Comparative Approaches, The Classical Press of Wales, Swansea, 2009, p. 48-59.

4 J. Peletier, Les oeuvres poétiques, Paris, Michel de Vascosan, 1547, p. 7-37. Cette œuvre 
L'attrait des auteurs des $\mathrm{XVI}^{\mathrm{e}}$ et $\mathrm{XVII}{ }^{\mathrm{e}}$ siècles pour les odes grecques témoignait d'un intérêt esthétique, mais aussi religieux ${ }^{5}$ et politique. L'esthétique d'Homère connut plusieurs imitations dans le courant du XVI siècle et fit l'objet un engouement important à mesure que les Français s'aventuraient à la conquête du Nouveau Monde. Les balbutiements du colonialisme français mirent en effet les premiers explorateurs et colons en présence de situations, de paysages, de faunes et surtout de personnes encore inconnues, qu'il fallait chercher à appréhender. Par ailleurs, ces tentatives de compréhension de phénomènes nouveaux sont à mettre en parallèle avec une autre mode littéraire, qui s’inspirait des œuvres homériques ou virgiliennes ${ }^{6}$ pour exalter la beauté de la nature sauvage ${ }^{7}$.

La poésie pastorale, très populaire dans les milieux artistiques (littéraires, picturaux ou architecturaux) de l'époque, renvoie à l'Arcadie antique comme modèle de beauté naturelle, primitive et sauvage, voire, dans certains cas, de fertilité ${ }^{8}$. L'historiographie fait remonter cette mode à l'Arcadia de Jacopo Sannazzaro, publiée en 1504, qui s'inspire des œuvres bucoliques romaines, comme les Bucoliques ou les Géorgiques de Virgile?. Ce mouvement connait par ailleurs son apogée à la fin du XVII siècle, avec la fondation de l'académie d'Arcadie de Rome $^{10}$. Cette association littéraire agissait avec la bénédiction du pape et s'inscrivait dans une ligne contre-réformiste marquée, où le pastoralisme devenait un outil de propagande au service du catholicisme" ${ }^{11}$. D'une manière générale, la mode pastorale et l'image de l'Arcadie renvoient à l'évocation d'un âge d'or, mélange d'abondance, de prospérité et de simplicité primitive que l'homme

précoce de Peletier incluait également une traduction des Géorgiques de Virgile. Peletier n'avait toutefois édité que les deux premiers livres de l'Odyssée.

5 N. Hepp, “ Les interprétations religieuses d'Homère au XVII ${ }^{\mathrm{e}}$ siècle », Revue des sciences religieuses, 21 (1957), p. 34-50. La théologie d'Homère et ses inspirations potentielles firent l'objet de diverses théories, allant de l'illumination divine à un plagiat des textes hébraïques.

6 H. Westra, “ Références classiques implicites et explicites dans les écrits des Jésuites sur la Nouvelle-France », Tangence 92 (2010), p. 27-37.

7 M.-Ch. Pioffet, La tentation de l'épopée dans les relations des jésuites, Sillery, Septentrion, 1997, p. 82-84. Voir aussi A. Grafton, New worlds, ancient texts. The power of tradition and the shock of discovery, Cambridge, Mass. - Londres, Harvard University Press, 1992, p. 61-93.

8 Voir notamment l'Aminta du Tasse (1573) ou l'Arcadia de Philip Sidney (1593).

9 M. Deramaix, “Ubi est Arcadia ? », Revue Fontenelle, 10 (2013), p. 17-22 et A.R. Ruff, Arcadian visions, Oxford - Havertown, Windgather Press, 2015, p. 1-14.

10 M.T. Graziosi, L'Arcadia. Trecento anni di storia, Rome, Palombi, 1991, p. 9-10.

11 G. Compagnino \& G. Savoca, Dalla vecchia Italia alla cultura europea del Settecento, Rome - Bari, Laterza, 1973, p. 48. 
a depuis longtemps perdu et qu'il cherche à retrouver. Il s'agit d'une variante terrestre du jardin d'Éden ${ }^{12}$. Dans la littérature française, cette image de liberté et d'abondance allait connaître un usage important de la part des premiers colons du nouveau monde.

Les Français ont en effet fondé leurs premières colonies permanentes en Acadie et en Nouvelle-France au début du XVII ${ }^{e}$ siècle. Parmi les premiers colons se trouvaient quelques lettrés, religieux ou hommes de loi. Ces auteurs compilent et publient les premiers rapports sur les territoires colonisés, ainsi que sur les indigènes peuplant les alentours des colonies. Les premières rencontres d'indigènes avaient initié un discours encore inédit, qu'Andreas Motsch assimile à une première forme d'ethnographie ${ }^{13}$. La plupart de ces premières études véhiculent une image positive des indigènes, selon une logique relevant du concept du «bon sauvage » ${ }^{14}$. Cette notion, présente dans les récits de voyage depuis l'Antiquité, connait une diffusion importante dans les milieux humanistes du XVI ${ }^{e}$ siècle. Cela fut perceptible dans les années qui suivirent la conquête espagnole du Mexique, avec la parution d'ouvrages critiquant la politique coloniale des Européens ainsi que les traitements infligés par les colons aux indigènes, modèles de vertu et de liberté ${ }^{15}$. De ces premières entreprises colonisatrices naît aux XVI ${ }^{\mathrm{e}} \mathrm{XVII} \mathrm{I}^{\mathrm{e}}$ siècles un nouveau genre dans la littérature française, les relations de voyages, descriptions dramatisées des découvertes et des voyages par les premiers explorateurs ${ }^{16}$. Le nom sera par la suite utilisé comme intitulé pour les rapports annuels des jésuites. Parmi les premières relations, citons celles publiées par Samuel de Champlain :

12 L. Lerner, “ The pastoral world: Arcadia and the Golden Age », in B. Loughrey (dir.), The pastoral mode, Londres, Macmillan, 1984, p. 145-154 et S. Macé, L'Éden perdu. La pastorale dans la poésie française de l'âge baroque, Paris, Honoré Champion, 2002, p. 319-354.

13 E. Bouchard \& R. Ouellet, “Sauvages du Canada et du Pacifique chez Bougainville », Revista española des Estudios canadienses 3.2 (2000), 1997, p. 9 et A. Мотsсн, Lafitau et l'émergence du discours ethnographique, Paris-Québec, Septentrion, 2001, p. 263-264.

14 S. Cro, The Noble Savage, Waterloo, Wilfried Laurier University Press, 1989, p. 13-38. L'imagerie du bon sauvage influença également les missionnaires jésuites et récollets dès le début des entreprises d'évangélisation. J. WARwick, “ Humanisme chrétien et bons sauvages ", XVII siècle 97 (1972), p. 33.

15 À cet égard, l'exemple de Pierre Martyr mérite d'être cité. P. Martyr, De orbo novo VII, 4. Voir Cro, The Noble Savage, p. 33.

16 R. Ouellet, «Qu'est-ce qu'une relation de voyage? », in Cl. Duchet \& S. Vachon (dir.), La recherche littéraire, objets et méthodes, Montréal - Paris, XYZ éditeur - Presses universitaires de Vincennes, 1993, p. 235-252. 
« Du costé de l'Est est une isle qui s'appelle Sainct Laurens, où est le Cap-Breton, $\&$ où une nation de sauvages appelez les Souricois hyuernent. Passant le destroit de l'isle Sainct Laurens, costoyant la coste de l'Arcadie, on vient dedans une baye qui vient ioindre ladite mine de cuiure. Allant plus outre, on trouve une rivière qui va quelques soixante et quatre vingts lieuës dedans les terres, laquelle va proche du lac des Irocois, par où lesdicts sauuages de la coste d'Arcadie leur vont faire la guerre. „ 17

L'auteur, fondateur des premières habitations à Port-Royal et à Québec, baptise d'abord “ Arcadie » la terre découverte en 1603, mais modifie le nom pour “Acadie » dès l'Année suivante ${ }^{18}$. Cette référence pose la question de l'ampleur de ses connaissances sur l'Antiquité classique, question d'autant plus délicate que l'on ignore tout de son éducation ${ }^{19}$. Cette référence à l'Arcadie peut impliquer qu'il ait voulu intégrer sa découverte dans un registre antiquisant, bien qu'elle ait pu également résulter d'une déformation d'un nom amérindien, voire d'une erreur d'orthographe de la part de Champlain puisqu'il ne recourut plus au terme “Arcadie » par la suite ${ }^{20}$. Il pouvait également s'agir d'une référence artistique de Champlain, à une époque où l'Arcadie, modèle de territoire sauvage et poétique, constituait une inspiration récurrente pour différents artistes. Cette dernière hypothèse demeure vraisemblable, au point qu'elle permettrait de conclure à une certaine richesse culturelle de Champlain, au contraire de ce qu'avançait David Fisher qui soutient l'idée d'une faible formation classique de l'auteur ${ }^{21}$. Marcel Trudel attribue le premier emploi du mot “ Arcadie » à Verrazano, qui s'en serait servi pour baptiser l'actuelle Virginie. Par la suite, les cartographes auraient fait remonter ce nom jusqu'en actuelle Acadie ${ }^{22}$, jusqu'à ce que Champlain le récupère pour baptiser sa découverte. Les raisons qui l'ont par la suite poussé à modifier de nouveau ce nom pour «Acadie» demeurent méconnues, ce qui entretient l'idée d'une erreur de l'auteur, même si ce dernier a pu agir ainsi afin d'éviter la confusion ou par volonté de simplification. Il est cependant peu probable que le recours à l'Arcadie ne relève que de la seule coïncidence à une époque où elle est une importante source d'inspiration poétique. En effet, son utilisation permet de

17 S. de Champlain, Euvres, texte édité par C.-H. Laverdière, Québec, G.E. Débarats, 1870 , p. 51.

18 S. de Champlain, Des Sauvages, Texte établi, présenté et annoté par A. Beaulieu et R. Ouellet, Montréal, L’Hexagone, 1993, p. 81.

19 Sur l'éducation de Champlain, l'historiographie est réduite à des supputations. Il fut peut-être baptisé comme un protestant, reçut une éducation protestante et se convertit ensuite au catholicisme. D.H. Fisher, Champlain's dream, New York - Londres, Simon \& Schuster 2008, p. 24-25.

20 Champlain, Euvres, p. 51, n. 3.

21 Fisher, Champlain's dream, p. 24-25.

22 M. Trudel, Histoire de la Nouvelle-France, Montréal, Fides, 1963, p. 62. 
déduire que le fondateur de Québec possédait des notions d'Histoire antique, qu'il était au fait des modes littéraires de son temps et qu'il était disposé à récupérer à son profit une confusion entretenue par les cartographes depuis près d'un siècle. Cette référence antique est également perceptible dans les illustrations des cartes que Champlain fit publier en France en $1613^{23}$.

Parmi les fondateurs du Canada, d'autres lettrés s'inspirèrent de la poésie antique. L'avocat Lescarbot est connu pour sa pièce Le théâtre de Neptune en la Nouvelle-France, soi-disant écrite et jouée à Port-Royal durant l'automne $1606^{24}$, mettant en scène le dieu Neptune et ses tritons et relatant leurs interactions avec des Amérindiens. On peut s'interroger sur l'existence de cette représentation qui s'accorde mal avec les circonstances difficiles de la fondation de l'Acadie et sur le peu de connaissances que les marins pouvaient avoir de l'Antiquité. Seule sa publication en Europe au retour des explorateurs avait une réelle importance, puisqu'elle permit de diffuser à large échelle la vision que Lescarbot se faisait de l'entreprise. La pièce témoigne d'une pensée colonialiste, la France ayant intérêt à s'établir dans les territoires récemment explorés. L'auteur fait ainsi parler le troisième Triton en ces termes :

«France, tu as occasion

De loüer la devotion

De tes enfans dont le courage

Se montre plus grand en cet âge

Qu'il ne fit onc és siecles vieux,

Estants ardement curieux

De faire eclater tes loüanges

Jusques aux peuples plus etranges,

Et graver tou les immortel

Meme souz ce monde mortel.

Aide doncques \& favorise

Une si loüable entreprise.

Neptune s'offre à ton secours

Qui les tiens maintiendra toujours

Contre toute l'humaine force,

23 M.J. Mckay, Picturing the land. Narrating territories in Canadian landscape art, 15001950, Montreal-Kingston, McGill-Queen's University Press, 2011, p. 21-24. L'auteur établit un parallélisme entre les gravures illustrant les Amérindiens sur les cartes de Champlain et les statues de l'Antiquité. Sur l'idéologie du noble sauvage dans la littérature coloniale, voir aussi T.J. ElLingson, The myth of the noble savage, Berkeley, University of California Press, 2001, p. 21-22.

24 P. CARILE, Le regard entravé. Littérature et anthropologie dans les premiers textes sur la Nouvelle-France, Sillery, Septentrion, 2000, p. 83-85. 
Si quelqu'un contre toy s'efforce.

Il ne faut jamais rejetter

Le bien qu'un Dieu nous veut preter. „25

Lescarbot agissait dans une logique de contre-réforme. Il revint en France avec une perception positive des terres américaines et de leurs habitants. Les Amérindiens eux-mêmes étaient destinés à la conversion au catholicisme et à devenir sujets de la couronne de France ${ }^{26}$. Lescarbot fait parler un sauvage en ces termes :

“ De la part des peuples sauvages

Qui environnent ces païs

Nous venons rendre les homages

Deuz aux sacrées fleur-de-lis

Es mains de toy, qui de son Prince

Représente la Majesté

Attendans que cette province

Faces florir en piété,

En mœurs civils, \& toue chose

Qui sert à l'etablissement

De ce qui est beau et repose

En un royal gouvernement. ' ${ }^{27}$

Cette œuvre présente l'idéologie du bon sauvage dans un discours impérialiste $^{28}$. L'auteur défend la nécessité du pouvoir français à s'établir sur ces terres, au nom des bienfaits que les indigènes pourraient apporter aux Européens et de l'enrichissement réciproque que pourrait représenter une telle entreprise. Le théâtre de Neptune était une œuvre de propagande en faveur de la monarchie, dans laquelle l'auteur se permettait toutefois une série de recommandations aux autorités de son pays ${ }^{29}$. Paolo Carile a vu dans cette pièce un emprunt baroque destiné à rappeler le faste des joyeuses entrées des souverains dans les villes

25 M. Lescarbot, Les muses de la Nouvelle-France, Paris, Iban Millot, 1609, p. 14-15.

26 É. Thierry, Marc Lescarbot. Un homme de plume au service de la Nouvelle-France, Paris, Honoré-Champion, 2001, p. 92-112 et B. Emont, Marc Lescarbot: Mythes et rêves fondateurs de la Nouvelle-France, Paris, L'Harmattan, 2002, p. 246-256.

27 Lescarbot, Muses, p. 17.

28 Lescarbot admirait les coutumes des Amérindiens, notamment la chasse à laquelle tous les hommes s'adonnaient, alors qu'il s'agissait en France d'un privilège réservé à l'Aristocratie. M. Lescaвot, Voyages en Acadie (1604-1607) suivis de La description des mours souriquoises comparées à celles d'autres peuples, édition critique de M.-Ch.Pioffet, Paris, PUPS, 2007, p. 398-409 et Ellingson, Myth, p. 21 et suiv.

29 J. Wasserman, Spectacle of Empire: Marc Lescarbot's Theatre of Neptune in New France, Vancouver, Talon, 2006, p. 25. 
de France ${ }^{30}$. Ce spectacle aurait été conçu comme une simulation de prise de pouvoir sur les terres de Nouvelle-France ${ }^{31}$. En effet, le personnage de Neptune fit déjà partie de la symbolique déployée par Charles IX lors de ses entrées ${ }^{32}$. La terminologie qui y est employée témoigne d'une influence des odes de la poésie grecque. L'auteur présentait par ailleurs une autre de ses compositions, les Muses de la Nouvelle-France comme une ode pindarique dédiée au roi.

“ Neptune, donne moy des vers

Propres à resonner la gloire

Du plus grand Roy que l'univers

Ait produit de longue mémoire.

Et puis que sur tes moites eaux

Tendent leurs ailes noz vaisseaux,

Fay qu'avec eux ore ie vole. » ${ }^{33}$

L'ouvrage recourt à une poésie inspirée des odes antiques pour vanter la colonisation dans une série d'ouvrages de propagande. La thématique de l'identification aux grands voyages d'exploration de l'Antiquité est manifeste chez Lescarbot. Ce faisant, l'auteur fait remonter la colonisation française à un processus plus large, datant des origines de l'Histoire antique, bien que, dans certaines circonstances, il ait montré des scrupules à comparer sa propre entreprise à la mythologie antique. Faisant le récit d'une traversée périlleuse, dont il attribue le succès à l'intervention divine, l'auteur conclut en disant :

“ Après beaucoup de périls (que je ne veux comparer à ceux d'Ulysse, ni d'Énée pour ne souiller nos voyages saints parmi l'impureté) le sieur de Poutrincourt arriva au Port-Royal le quatorzième de Novembre. »34

30 Carile, Regard, p. 89-93 ; Wasserman, Spectacle, p. 25-28 et N. Hepp, “ Homère en France au XVI ${ }^{\mathrm{e}}$ siècle », Atti della Reale Accademia delle Scienze di Torino, 96 (1961-62), p. 85. Voir aussi N.-Fr. WAGner, “Écrire le roi au seuil de l'âge classique : pouvoir et fiction des entrées royales. De quelques fausses entrées » in J.-V. Blanchard \& H. Visentin (dir.), L'invraisemblance du pouvoir. Mise en scène de la souveraineté au XVII siècle, Paris, PUPS, 2005, p. 137-160.

31 Fr. Lestrignant, “Écrire la Nouvelle-France en 1609. Note sur Marc Lescarbot », in A. Beaulieu \& S. Chaffray (dir.), Représentation, métissage et pouvoir, Québec, Presses de l'université de Laval, 2012, p. 162-166.

32 Wasserman, Spectacle, p. 25-28.

33 Lescarbot, Muses, p. 1.

34 Lescarbot, Voyages, p. 203. 
Le recours à l'Histoire antique était également destiné à effacer les différences et à permettre d'appréhender la nouveauté. Dans le chapitre XIV de son Histoire de la Nouvelle-France, Lescarbot dédie un chapitre aux populations souriquoises, décrivant les différentes facettes de leur civilisation (religion, vêtements, mariage, chasse et pêche, guerre...).

“ La danse est une chose fort ancienne entre tous les peuples. Mais fut premièrement faite \& instituée ès choses divines, comme nous en venons de remarquer un exemple : \& les Canaéens qui adoraient le feu faisaient des danses alentour \& lui sacrifiaient leurs enfants, Or la façon de danser n'était de l'invention des idolâtres, ains du peuple de Dieu. Car nous lisons au livre des Juges qu'il y avait une solennité à Dieu en Silo, où les filles venaient danser au son de la flûte. Et David faisant ramener l'Arche de l'alliance en Jérusalem allait devant en chemise, dansant de toute sa force.

Quant aux Païens ils ont suivi cette façon. Car Plutarque en la vie de Nicias dit que les villes Grecques avaient tous les ans coutume d'aller en Délos célébrer les danses \& chansons à l'honneur d'Apollon. Et en la vie de l'Orateur Lycurge le même dit qu'il en institua une fort solennelle au Pirée à l'honneur de Neptune, avec un jeu de prix de la valeur au mieux dansant, de cent écus, à l'autre d'après de quatre-vingts, \& au troisième de soixante. »35

Si Lescarbot ne fait pas d'allusion directe à la colonisation grecque, sa description des colonies françaises est empreinte de références à l'Histoire grecque. Le recours à l'Histoire des cités grecques offre un parallèle facile avec une population aux mœurs jugées primitives et permet une comparaison des nouvelles découvertes avec un modèle plus familier. En outre, certaines figures mythologiques, comme celles issues de l'épopée homérique, renvoie à une forme primitive d'exploration coloniale ou de rapport de voyage. L'idée que l'avocat se fait d'un chef d'expédition en est une bonne illustration.

“ Il n'appartient pas à tout le monde de conduire des peuplades et des colonies. Qui veut faire cela il faut qu'il soit populaire et de tous metiers, et qu'il ne se dedaigne de rien : et sur tout qu'il soit doux et affable, et éloigné de cruauté. » ${ }^{36}$

Ces qualités d'un chef trouvent un écho dans la préface du chapitre consacré aux mœurs souriquoises, où l'auteur fait référence aux qualités d'un personnage mythique comme Ulysse.

35 Lescarbot, Voyages, p. 355-356.

36 M. Lescarbot, Histoire de la Nouvelle France, Paris, Iban Millot, 1611, p. 228. Voir Fr. Lestrignant, “Champlain, Lescarbot et la "conférence” des histoires », in N. Doiron (éd.), Scritti sulla Nouvelle-France nel Seicento, Bari, Adriatica, 1984, p. 80. 
“ Or d'autant que c'est peu de chose de savoir que des peuples sont différents de nous en mœurs \& coutumes, si nous ne savons le particularités d'icelles : peu de chose aussi de ne savoir que ce qui nous est proche : ains est une belle science de connaitre la manière de vivre de toutes les nations du monde, pour raison de quoi Ulysse a été estimé d'avoir beaucoup vu et connu. » ${ }^{37}$

À travers Homère, l'avocat exalte l'initiative et l'entreprise individuelles, notamment contre les religieux, qu'il accusait de vouloir dénaturer les pratiques des indigènes au nom des impératifs de la christianisation ${ }^{38}$.

D'autres témoignages firent écho à ceux de Lescarbot à mesure que les missionnaires arrivèrent en Nouvelle-France pour évangéliser les Amérindiens. Dans un premier temps, les missions sont réparties entre les membres de la Compagnie de Jésus et les Récollets, ordre issu du mouvement franciscain, qui prônait une observance stricte de la règle de Saint-François ${ }^{39}$. Le récollet Gabriel Sagard se sert de l'Histoire des cités antiques pour traiter des mœurs politiques des Hurons, notamment l'autorité des assemblées et leur influence dans les déclarations de guerre ${ }^{40}$. Partant d'une épître de Pline le Jeune à Fabatus, le religieux se penche sur le rôle des anciens dans les décisions politiques de la cité de Maserde en Achaïe ${ }^{41}$, comparant la situation de cette cité avec celle des villages hurons.

“ Pline, en une Epitre qu'il escrit à Fabate, dict que Pyrrhe, Roy des Epirotes, demanda à un philosophe qu'il menoit avec lui, quelle estoit la meilleure Cité du monde. Le philosophe respondit, la meilleure Cité du monde, c'est Maserde, un lieu de deux cens feux en Achaye, pour ce que tous les murs sont de pierres noires, \& tous ceux qui la gouvernent ont des testes blanches. Ce philosophe n'a rien dit (en cela) de luy-mesme : car tous les anciens, apres le Sage Salomon, ont dit qu'aux vieillards se trouvoit la sagesse : \& en effect, on voit souvent la ieunesse d'ans, estre accompagnee de celle de l'esprit. Les capitaines entre nos Sauvages, sont ordinairement plutost vieux que ieunesm \& viennent par succession, ainsi que la Royauté par deçà, ce qui s'entend, si le fils d'un capitaine enfuit la vertu du père ; car autrement ils font comme aux vieux siècles, lors que premierement ces peuples efleurent des Roys : mais ce Capitaine n'a point entr'eux authorité absoluë, bien qu'on luy ait quelque respect, \& conduisent le

37 Lescarbot, Voyages, p. 341.

38 Lescarbot, Histoire de la Nouvelle-France, p. 670. Voir G.-A. Vachon, "Primitifs canadiens », Études françaises 4.1 (1968), p. 59.

39 C. Galland, Pour la gloire de Dieu et du Roi. Les récollets en Nouvelle-France aux XVII et

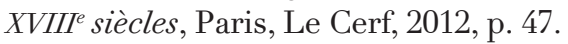

40 G. SAgard, Le grand voyage du pays des Hurons, Paris, Denys Moreav, 1632, p. 196-224.

41 L. Calpurnius Fabatus, chevalier romain, grand-père de la troisième épouse de Pline le Jeune qui a entretenu avec lui une correspondance qui nous est en partie parvenue. Pline le Jeune IV, 1 ; V, 11 ; VI, 12 et 30 ; VII, 11, 16, 23 et 32 et VIII, 10. 
peuple plustost par prieres, exhortation, \& par exemple, que par commandement. Le gouvernement qui est entr'eux est tel que les anciens et les principaux de la ville ou du bourg s'assemblent en un conseil avec le Capitaine, où ils decident \& proposent tout ce qui est des affaires de leur Republique, non par un commandement absolu, comme i'ay dict ; ains par supplications \& remontrances, \& par pluralité des voix qu'ils colligent, avec de petits fetus de joncs. » ${ }^{42}$

Le récollet insiste sur l'importance d'un contre-pouvoir, symbolisé chez les Hurons par une assemblée de citoyens plus fortunés. Cette assemblée avait la possibilité de contrer les éventuelles mauvaises décisions d'un chef. Cela permit à Jack Warwick d'affirmer que Sagard estimait davantage les assemblées de citoyens, identiques à celles des cités antiques qu'un régime dirigé par un ancien ${ }^{43}$. L'extrait mentionné par Sagard n'apparaît cependant pas chez Pline, pas plus que la cité de Maserde et il est vraisemblable qu'il s'agissait d'une invention destinée à soutenir sa théorie. Warwick attire ainsi l'attention sur l'usage que fait Sagard des sources anciennes, là où les Jésuites, pourtant formés aux lettres antiques, ne les utilisaient pas encore pour leurs comparaisons avec les Amérindiens. Warwick attribue cet état de fait à une volonté de Sagard de rapprocher les différents peuples, là où les Jésuites insistaient davantage sur la supériorité de l'homme civilisé ${ }^{44}$. Du point de vue de la Compagnie de Jésus, il faudra en effet attendre Lafitau au début du XVIII siècle pour comparer les Iroquois aux anciens Grecs.

À la même époque, le Jésuite Pierre Biard, comparait les péripéties des navigateurs français à celles d'Ulysse.

“ La peine estoit de trouver quelque bon Aeole, Roy des Autans Bursins, qui les voulust donner non comme ils le furent à Ulysses, liés dans le cuir pour ne souffler pas, aons deliés et de bon cours pour bouffer dans les voiles, car sans cela point de navire ne sçauroit avancer. » ${ }^{45}$

Un autre jésuite, Paul Lejeune, premier supérieur de l'ordre au Canada, fut en charge des Relations des jésuites de 1632 à 1642. Il se sert d'une série de topoi pour décrire les autochtones ou les colons ${ }^{46}$. Son œuvre laisse une place importante à l'image et au vocabulaire militaire, que ce soit pour décrire la colonisation ou la conversion des Amérindiens. L’auteur vante les mérites des soldats français, qui

42 SAGARD, Grand voyage, p. 196-197.

43 Warwick, Humanisme, p. 30-32.

44 J. Warwick, "L’Antiquité dans le cadre référentiel du "sauvage" : 1615-1642 », in G. Thérien (dir.), Figures de l'Indien, Montréal, Université du Québec, 1995, p. 126.

45 Relations des Jésuites, tome 1, Québec, 1858, p. 38.

46 K.-D. ErtLer, "Les Relations des Jésuites face au monde indigène », in M.A. Bernier et alii (dir.), Jesuit accounts of colonial Americas. Intercultural transfers, intellectual disputes, and textualities, Toronto, University of Toronto Press, 2014, p. 285. 
refusent de fuir et affrontent un ennemi supérieur en nombre, au contraire des indigènes dont il fustige la lâcheté.

“ Nos François ne vouloient point aller avec eux, pour ce, disent-ils, que les sauvages ne sçauroient obeïr, ny tenir pied ferme en guerre ; à la premiere fantaisie ils s'envolent comme des oyseaux, si bien qu'il faudroit aussi que nos François, estans un petit nombre, prissent la fuitte, ce qui les rendroit fort honteux, car on se moque parmy nous des fuyards. Les braves soldats, tels que nous icy, veulent vaincre ou mourir. ${ }^{\mathbf{4 7}}$

Marie-Christine Pioffet a restreint les inspirations de l'œuvre de Lejeune à la seule terminologie homérique ${ }^{48}$. S’il est vrai que le Jésuite s'est inspiré d'une littérature héroïque pour traiter des premières guerres coloniales et de la survivance de la Nouvelle-France, il puisait aussi dans la littérature historique pour décrire les autochtones ${ }^{49}$. L'héroïsme chez Lejeune est le plus souvent l'apanage des Français, bien que les Relations des années ultérieures aient transmis une image positive des Amérindiens convertis. Les Relations de 1644 décrivent un noble amérindien converti et devenu missionnaire sur ses terres en des termes favorables, inspirés de la représentation antique.

“ En un mot, il est de ces personnes qui portent sur le front ie ne sçay quoy de digne d'empire, et à le voir un arc ou une espée en main, on diroit que c'est un portait de ces anciens Cesars dont nous ne voyons en Europe que des images toutes enfumées : la foy en a fait un excellent chrestien. „50

Pour les Amérindiens hostiles aux Français, les auteurs des Relations se serviront à nouveau de références antiques pour insister sur leur différence ou illustrer leur cruauté.

“On le trouve estendu sur la terre, transpercé de coups d'espée, trempé dans son sang, et desia un petit endommagé du bec des oiseaux. La guerre des Sauvages n'est non plus la guerre des François, que la guerre des Parthes n'estoit point la guerre des Romains. ” ${ }^{51}$

47 ERTLER, Relations, p. 61-62.

48 M.-Ch. Pioffet, “ L’arc et l'épée : les images de la guerre chez le jésuite Paul Lejeune » in R. Ouellet \& A. Beaulieu (dir.), Rhétorique et conquête missionnaire : le jésuite Paul Lejeune, Sillery, Septentrion, 1993, p.41-45.

49 M. Parent, "Restriction de validité et héroïsation du protagoniste dans Le Grand voyage du pays des Hurons de Sagard et la Relation de 1634 de Lejeune », in Ouellet \& Beaulieu (dir.), Rhétorique et conquête, p. 67-87.

50 B. Vimont, Relations des Jésuites contenant ce qui s'est passé de plus remarquable dans les missions des pères de la Compagnie de Jésus dans la Nouvelle-France, tome 2 (1643-1644), Paris, Sébastien Cramoisy, 1645, p. 96.

51 Vimont, Relations, p. 19. 
Enfin, le récollet Chrestien Le Clercq, à la fin du XVII ${ }^{\mathrm{e}}$ siècle, comparait la tenue des Hurons à celle d'Hercule après avoir tué le lion de Némée.

“Quoique quelques-uns de nos sauvages se servent aujourd'huy de couvertures, capots, just'au-corps, \& des étofes qu'on apporte de France pour leur faire des habits ; il est toutefois constant qu'avant l'établissement des François dans ce nouveau Monde, ils ne se couvroient que de peaux d'orignac, de castors, de martes \& de loups marins, dont sont encore à presens vêtus plusieurs de ces peuples. La figure \& la representation d'Hercules, qui a sur ses épaules en forme de manteau, la peau du lion qu'il avoit genereusement vaincu \& terrassé, comme l'Histoire rapporte, est à peu près celle d'un Sauvage dans sa cabanne, vêtu à la mode de ses ancêtres... " ${ }^{52}$

L'objectif des religieux était d'intégrer cet ensemble de peuples nouvellement soumis à l'universalisme catholique, processus d'autant plus indispensable que certains Jésuites, comme Lejeune, étaient convaincus du déisme des Amérindiens ${ }^{53}$. Par ailleurs, si Lejeune établit une forte différence entre les indigènes et les Européens, ce mode de pensée n'était pas partagé par tous les Jésuites, au contraire de ce que pensait Warwick ${ }^{54}$.

Les rapports des premiers explorateurs de la Nouvelle-France accordent une part importante aux récits de voyage. Le recours à une personnalité comme Ulysse, symbole du héros voyageur et inventif, était fréquent dans la littérature du début du XVII siècle. Il est établi que les odes antiques servaient de comparaison destinée à permettre au public européen d'appréhender les nouvelles découvertes et à en vanter les intérêts. Ainsi, la comparaison à l'Arcadie, qui constitue une mode courante aux Temps modernes, s'inscrit dans une propagande destinée à inciter d'éventuels investisseurs à s'impliquer dans le processus colonial ${ }^{55}$. Néanmoins, l'utilisation de l'Histoire non-mythique de l'Antiquité par les auteurs du début du XVII ${ }^{\mathrm{e}}$ siècle dénote une contextualisation du colonialisme, à une époque où les colonies françaises tardaient à se développer. Les auteurs se focalisaient sur le voyage en tant que processus mais également sur les régimes politiques

52 Ch. Le Clerce, Nouvelle relation de la Gaspésie, Paris, A. Auroy, 1691, p. 52-53.

53 D. Deslandres, “Le jésuite, l'“intoléré" et le "sauvage". La fabrication par omission d'un mythe », in Ch. Grell \& Ch. Michel (dir.), Primitivisme et mythes des origines dans la France des Lumières, 1680-1820, Paris, PUPS, 1988, p. 98-99; S.E. Melzer, Colonizer or Colonized: The Hidden Stories of Early Modern French Culture, Philadelphie, University of Pennsylvania Press, 2012, p. 106-109.

54 Les réticences de Lejeune à l'égard des Amérindiens étaient en partie dues à l'échec de ses propres tentatives pour les évangéliser. Y. LE BRAs, “L'Autre dans les Relations de Paul Le Jeune » in Thérien (dir.), Figures de l'Indien, 1995, p. 166-167.

55 Mckay, Picturing the land. p. 21-24. 
appliqués par les indigènes, régimes pouvant présenter certaines qualités aux yeux des observateurs (comme le contre-pouvoir des assemblées chez Sagard). Ce type de littérature vante la colonisation en ne se fondant que sur des critères esthétisants, ce qui devint insuffisant pour les ordres religieux qui suivirent les premiers explorateurs.

Le processus colonial tel qu'initié par les Français impliquait une intégration des territoires mais aussi des populations autochtones, sujets susceptibles d'être soumis à la foi catholique, voire, pour certains auteurs, aux mœurs françaises ${ }^{56}$. La francisation des Amérindiens comme mécanisme destiné à faciliter leur conversion est cependant plus fréquente chez les Récollets que chez les Jésuites ${ }^{57}$. Une personnalité comme Lejeune avance au contraire que la civilisation condamnerait les Amérindiens à la décadence ${ }^{58}$. Cette position, proche de celle de Lescarbot, contribue à expliquer le recours à la mythologie, pour attester du déisme des Amérindiens, selon un mode de pensée qui sera celui d'un Lafitau un siècle plus tard. En effet, ce dernier prétend que les Amérindiens pouvaient être assimilés aux anciens Grecs et connaîtraient par conséquent un parcours religieux similaire ${ }^{59}$. En recourant aux odes antiques, Lescarbot et les missionnaires s'inscrivent dans le processus d'emploi politique de l'Antiquité initié depuis le XVI siècle ${ }^{60}$, phénomène observé chez Sagard et sa description des mœurs politiques des Hurons. Toutefois, l'essentiel des œuvres citées renvoie au colonialisme comme phénomène nouveau davantage qu'à des notions politiques comme la polis dont la récupération aux Temps modernes a été décrite par Hartog ${ }^{61}$. La redécouverte de l'Odyssée et son utilisation rhétorique aux prémices de la colonisation française marque le début d'une littérature de propagande qui outrepassa le seul contexte religieux (attaché à l'esprit missionnaire et martyr) ${ }^{62}$ ou politique pour défendre

56 Notamment par le métissage, envisagé très tôt. G. Havard, “"Nous ne ferons plus qu'un peuple”. Le métissage en Nouvelle-France à l'époque de Champlain », in G. Martinière \& D. Poton (dir.), Le Nouveau-Monde et Champlain, Paris, Les Indes savantes, 2008, p. $89-110$.

57 D. Deslandres, Croire et faire croire. Les missions françaises au XVII e siècle (1600-1650), Paris, Fayard, 2003, p. 241-261.

58 Deslandres, Jésuite, p. 98-99.

59 Мотsch, Lafitau, p. 85.

60 Notamment par Machiavel. N. Machiavelli, Discorsi sopra la prima decade di Tito Livio, Florence, Bernardo di Giunta, 1531, livre I, chap. 4. Voir Fr. Hartog, Anciens, Modernes, Sauvages, Paris, Galaade, 2005, p. 170-176.

61 Hartog, Anciens, p. 170-176. Voir aussi Br. Seitz \& Th. Thorp, The Iroquois and the Athenians. A political ontology, Plymouth, Rowman \& Littlefield, 2013, p. 1-15.

62 M.-Ch. Pioffet, « La Nouvelle-France dans l'imaginaire jésuite », in Bernier et alii (dir.), Jesuit accounts, p. 337-338. 
une vision pragmatique et temporelle du colonialisme tel qu'il sera pratiqué par la France dans les années qui suivirent.

\section{Nicolas Faelli}

Ph. D.

Université de Montréal

Université libre de Bruxelles

1100, Boulevard de Montarville,

Boîte 204, J4B-5Z1, Boucherville, Qc,

Canada

nfaelli@hotmail.com 